\title{
Nonequilibrium electron-vibration coupling and conductance fluctuations in a $\mathrm{C}_{60}$-junction
}

\author{
Søren Ulstrup,,$^{1, *}$ Thomas Frederiksen, ${ }^{2,3}$ and Mads Brandbyge ${ }^{1}$ \\ ${ }^{1}$ DTU-Nanotech, Department of Micro- and Nanotechnology, \\ Technical University of Denmark, Ørsteds Plads, \\ Bldg. 345E, DK-2800 Kongens Lyngby, Denmark \\ ${ }^{2}$ Donostia International Physics Center (DIPC) - UPV/EHU, E-20018 Donostia-San Sebastián, Spain \\ ${ }^{3}$ IKERBASQUE, Basque Foundation for Science, E-48011, Bilbao, Spain
}

(Dated: March 4, 2022)

\begin{abstract}
We investigate chemical bond formation and conductance in a molecular $\mathrm{C}_{60}$-junction under finite bias voltage using first-principles calculations based on density functional theory and nonequilibrium Green's functions (DFT-NEGF). At the point of contact formation we identify a remarkably strong coupling between the $\mathrm{C}_{60}$-motion and the molecular electronic structure. This is only seen for positive sample bias, although the conductance itself is not strongly polarity dependent. The nonequilibrium effect is traced back a sudden shift in the position of the voltage drop with a small $\mathrm{C}_{60}$-displacement. Combined with a vibrational heating mechanism we construct a model from our results that explain the polarity-dependent two-level conductance fluctuations observed in recent scanning tunneling microscopy (STM) experiments [N. Néel et al., Nano Lett. 11, 3593 (2011)]. These findings highlight the significance of nonequilibrium effects in chemical bond formation/breaking and in electron-vibration coupling in molecular electronics.
\end{abstract}

PACS numbers: 73.63.-b, 68.37.Ef, 61.48.-c

\section{INTRODUCTION}

The influence of an external bias voltage and electronic currents on the formation and breaking of chemical bonds is a topic of increasing importance with the continued down-scaling of electronic components. This is especially accentuated in the limit of single-molecule devices! 1

A substantial current may flow through a single bond and its effect on the stability and impact on transport is crucial. The phenomenon of random two-level conductance fluctuations (TLF) is generally observed in a wide range of simple atomic and molecular contacts. ${ }^{26}$ It is often possible to relate these to changes in the bonding configuration driven by the current. Clearly, controlled and reversible switching between well-defined conductance states is a useful function.$^{7}$ Over the years many examples of atomic ${ }^{6 / 819}$ and molecule-based $\frac{10}{22}$ switches have been demonstrated. However, the understanding of how the nonequilibrium electronic structure impact chemical bonding and conformational changes still pose many open questions. First-principles calculations and comparisons with well-characterized, time-resolved experiments can shed light on these issues.

Nonequilibrium dynamics of $\mathrm{C}_{60}$-systems has been under intense study $14 \mid 23$ 25. Here we focus on recently reported time-resolved measurements of single $\mathrm{C}_{60^{-}}$ contacts with a scanning tunneling microscope (STM) which showed that TLF occur in a narrow transition regime between tunneling and contact to $\mathrm{C}_{60}$. The advantage of STM is the possibility to identify the orientation of individual $\mathrm{C}_{60}$-molecules ${ }^{26-27}$ before and after controllable formation of the tip-molecule contact. ${ }^{28}$ Moreover, the role of detailed electrode bonding geometry 2 and contact point on the junction conductance has been clarified $\underline{31}$

More specifically, the experiment revealed the following interesting properties: (i) In the tunneling regime $d I / d V$ spectroscopy shows that transport is dominated by the lowest unoccupied molecular orbital (LUMO) (seen as a resonance centered at a positive sample voltage of $\sim 0.4 \mathrm{~V}$ ), while (ii) in contact the $I-V$ curve is close to linear in the voltage range $[-0.4 ;+0.4] \mathrm{V}$, suggesting a relatively symmetric coupling of the LUMO resonance to the two electrodes. Intriguingly, (iii) the TLF was only observed at positive sample voltage around contact formation. These findings were discussed in Ref. 25] solely on the basis of $d I / d V$ spectra in the tunnel regime. Essentially, only the spectral properties of the molecular adsorbate in equilibrium with the substrate were considered. Here we present a different view on the experimental findings based on our demonstration of a remarkably strong bias-dependent electronic coupling to the centerof-mass (CM) motion of the $\mathrm{C}_{60}$ at the point when a bond is being formed between $\mathrm{C}_{60}$ and the apex atom of the STM tip. From first-principles calculations we obtain a detailed description of the $\mathrm{C}_{60}$-junction geometry as well as the molecular LUMO resonance near the Fermi level. This allows us to construct a model for the TLF, which provides an explanation for the experimental findings. Our results demonstrate that the full nonequilibrium electronic structure needs to be accounted for to understand the observed TLF.

Our paper is organized as follows. In Sec. [II we describe the first-principles method and our setup of the $\mathrm{C}_{60}$-contact system. In Sec. III we then describe the results obtained without fitting parameters for the contact formation between STM-tip and $\mathrm{C}_{60}$ in equilibrium. Here we identify the formation of the chemical bond be- 


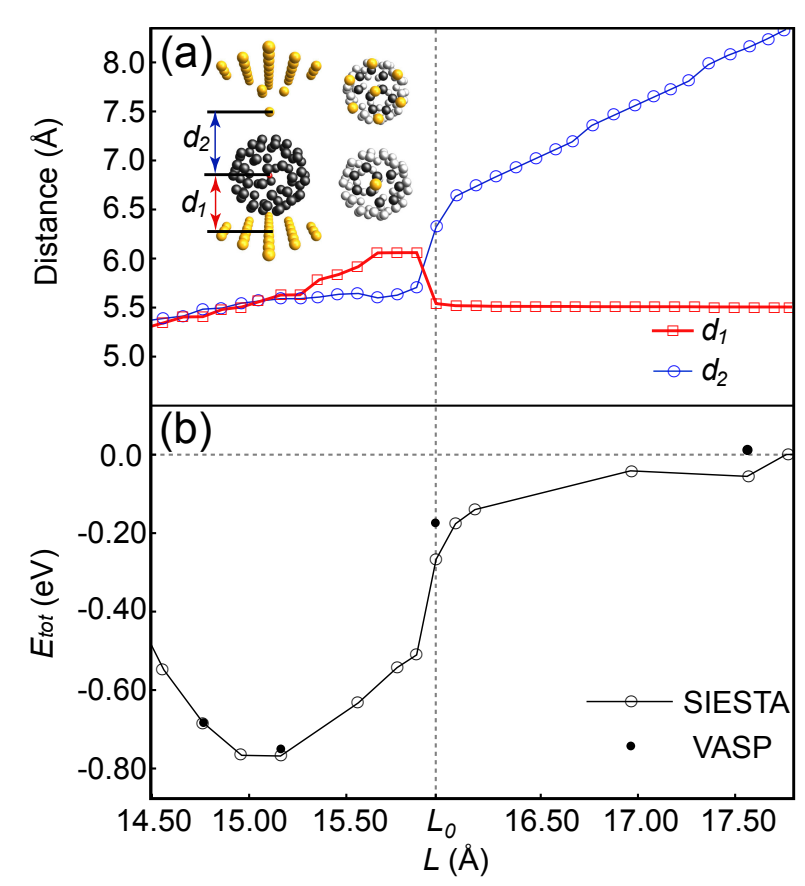

FIG. 1: (color online) (a) Relaxed bond lengths, and (b) corresponding total energy vs electrode separation $L$. The total energy is determined with respect to the initial configuration. The bond length $d_{1}\left(d_{2}\right)$ between $\mathrm{C}_{60}$ center-of-mass (CM) and surface (tip) and the junction geometry are defined in the inset in (a) along with the orientation of $\mathrm{C}_{60}$ with respect to the underlying $\mathrm{Cu}$ surface (top right inset) and the tip apex atom (bottom right inset). The $\mathrm{C}_{60}$ is oriented such that the tip apex atom approaches the 5:6 bond.

tween the molecule and the tip apex atom. This is followed by our study of nonequilibrium effects and a discussion of the identified polarity-dependent strong coupling between the $\mathrm{C}_{60} \mathrm{CM}$ motion and voltage drop (Sec. IV). From these first-principles calculations we extract in Sec. V $\mathrm{V}$ parameters for a simple single-resonance model, most importantly the bias-dependent electronvibration coupling to the CM motion. Together with a few additional parameters the model is used to calculate the TLF behavior, which can be compared to the experiment. Before concluding we discuss how the nonequilibrium forces modify the energy landscape for the CMmotion (Sec. VI).

\section{METHOD AND SETUP}

To study the contact formation and TLF we employ the SIESTA ${ }^{32}$ density functional theory (DFT) method, and its extension to finite bias using nonequilibrium Green's functions (DFT-NEGF) in the TRANSiESTA scheme ${ }^{33}$ The generalized gradient approximation (GGA-PBE) is applied for exchange and correlation $(\mathrm{xc}) ! 34$
The $\mathrm{C}_{60}$ junction geometry is modelled as shown in the inset to Fig. 1. The periodic supercell used in the DFT calculations contains one $\mathrm{C}_{60}$ molecule supported on top of seven fixed $\mathrm{Cu}(111)$ layers $(27 \mathrm{Cu}$ atoms per layer) with a pyramid-shaped $\mathrm{Cu}$ tip mounted on the bottom layer. To accurately describe the $\mathrm{Cu}$ surface and the chemical bonding with $\mathrm{C}_{60}$, an optimized diffuse basis set was applied for $\mathrm{Cu}$ surface layers and the tip $\stackrel{35}{\text { The }}$ counterpoise correction ${ }^{36}$ for the basis set superposition errors (BSSE) was applied to the total energy calculations, which was checked against complementary calculations with the $\operatorname{VASS}^{37}$ plane wave code as shown in Fig. 1(b).

The $\Gamma$-point approximation was employed for Brillouin zone integrations in the electronic structure calculation, while the transmission function was sampled over $3 \times 3$ k-points in the $2 \mathrm{D}$ Brillouin zone parallel to the electrode surfaces. The residual atomic forces were lower than $0.02 \mathrm{eV} / \AA$ for the atoms that were relaxed. The $\mathrm{C}_{60} \mathrm{CM}$ force constant was calculated from DFT total energies corresponding to configurations where the $\mathrm{C}_{60}$ $\mathrm{CM}$ was rigidly displaced, up to $0.6 \AA$ from its equilibrium position.

\section{CONTACT FORMATION}

We first focus on the bond-formation point at zero bias, and consider the approach of the STM tip towards a 5:6 $\mathrm{C}_{60}$-bond, i.e., a bond between a pentagon and a hexagon. We note that the fluctuations were observed for this orientation in the experiments, $\stackrel{25}{25}$ and that no molecular rotations occur during contact formation in either the experiments ${ }^{\sqrt{27}}$ or in our structure optimizations.

We optimize the junction geometry by stepwise reducing the size of the DFT-supercell in the direction perpendicular to the surface, while relaxing the $\mathrm{C}_{60}$ and tip atoms. Fig. 1(a) shows the relaxed bond lengths $d_{1}$ and $d_{2}$, between the $\mathrm{C}_{60}$ center-of-mass $(\mathrm{CM})$ and the surface and the tip atoms, respectively, as a function of electrode separation $L$. Around a characteristic separation $L_{0}=15.96 \AA$, the distance $d_{2}$ decreases rapidly while $d_{1}$ increases dramatically as the cell shrinks. This signals the onset of a chemical bond formation between the STM tip and the $\mathrm{C}_{60}$ molecule. This tip- $\mathrm{C}_{60}$ attraction lowers the total energy of the system as witnessed by the binding energy curve in Fig. 1.(b).

The corresponding vibrational energy $\hbar \Omega$ associated with the $\mathrm{C}_{60} \mathrm{CM}$ motion as well as the zero-bias conductance $G=\mathrm{G}_{0} T\left(E_{F}\right)$ (conductance quantum $\mathrm{G}_{0}=2 e^{2} / h$ ) of the junction are shown in Fig. 2 in the transition regime between tunneling and contact for the DFT equilibrium geometries. At $L_{0}$ we find three eigenchannels contributing to the total transmission $T\left(E_{F}\right)$ with the values $\{0.16,0.006,0.002\}$. The first channel dominates the transmission, because the three-fold degeneracy of the $\mathrm{C}_{60}$ LUMO has been lifted 38139 Thus, the $\mathrm{C}_{60}$ symmetry is broken in the contact configuration. One ob- 


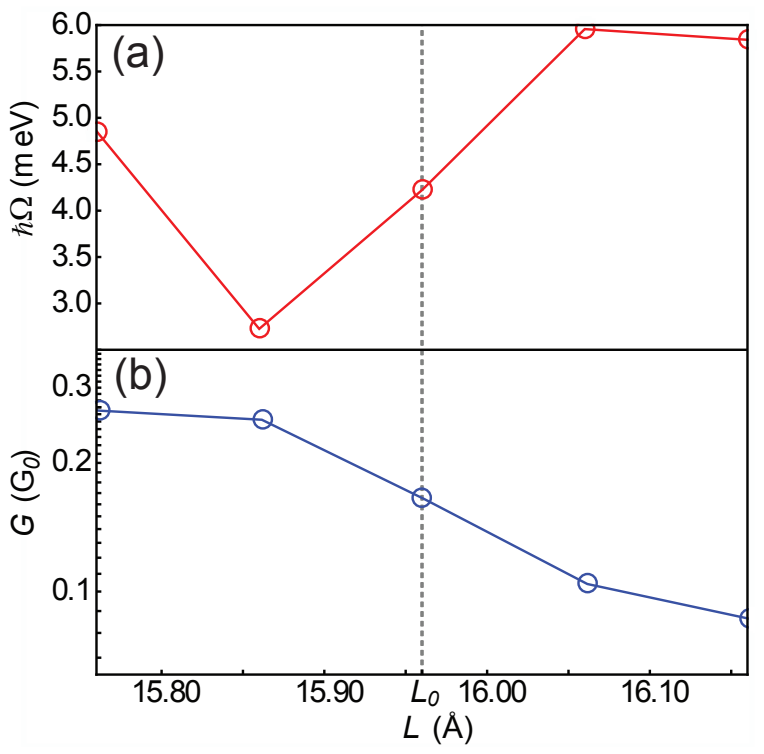

FIG. 2: (color online) (a) Vibrational energy $\hbar \Omega$ of the $\mathrm{C}_{60}$ center-of-mass (CM) motion between the electrodes, and (b) zero-bias conductance $G$ of the junction vs electrode separation $L$ in the transition regime between tunneling and contact for the DFT equilibrium geometries.

serves that the bond formation to the tip softens the $\mathrm{C}_{60^{-}}$ vibration [Fig. 2(a)] and increases the conductance by roughly a factor of 2.5 [Fig. 2(b)]. We note that the calculated conductance value of the order $G=0.2 \mathrm{G}_{0}$ agrees very well with the experimental conductance in the transition region between tunneling and contact where the TLF occur ${ }^{[25}$ Moreover, the calculated vibrational energies agree with a recent theoretical study of the $\mathrm{C}_{60}$ CM-motion on the $\mathrm{Au}(111)$ surface $\frac{40}{6}$

According to our equilibrium DFT calculations we could not identify two well-defined stable configurations (for any fixed electrode separation) which could explain the existence of two different conductance states. Instead we observe a shallow energy landscape around the point of contact formation indicating that $\mathrm{C}_{60}$ is rather free to move between the electrodes (e.g., the softening of the $\mathrm{C}_{60} \mathrm{CM}$ mode). We therefore speculate that a small barrier of the order of $10 \mathrm{meV}$, separating two distinct configurations, could be masked by limited numerical accuracy or by inherent approximations in the applied xc functional. In fact, recent theoretical studies of a somewhat simpler system consisting of graphene on $\mathrm{Ni}(111)$ have shown that various $\mathrm{xc}$ functionals can yield differences in the potential energies describing the carbonmetal distance much beyond the energies relevant for our system. $\stackrel{4142}{ }$ The disregard of current-induced forces acting on the atoms could also play an important role in the energy landscape, $\stackrel{43}{3}$ a point we return to at the end of this paper. Finally, we note that the actual experiments involve a complex reconstructed surface structure which we did not take into account. Because of these circumstances we shall therefore in our TLF-model (Sec. V) postulate the existence of two configurations in the contact region separated by a small barrier (on the order of DFT-accuracy), and instead focus our attention on the electron-CM vibration coupling and the resulting current-induced heating, which can explain the observed polarity-dependent TLF.

As the electrode separation $L_{0}$ is characteristic for the point of tip- $\mathrm{C}_{60}$ bond formation, we take this configuration as the starting point for an exploration of how the nonequilibrium electronic structure and electron transport depend on $\mathrm{C}_{60}$ motion. Fig. 3(a) shows the transmission spectra (with a prominent LUMO resonance) for several positions $\Delta d_{1}$ of $\mathrm{C}_{60}$ between the electrodes under three different applied sample voltages $V_{S}$. In each situation the transmission function is approximately given by a Breit-Wigner function $\underline{44 / 45}$

$$
T\left(E, V_{S}\right) \approx 2 \pi \frac{\Gamma_{T} \Gamma_{S}}{\Gamma_{T}+\Gamma_{S}}\left[\rho_{T}\left(E, V_{S}\right)+\rho_{S}\left(E, V_{S}\right)\right],
$$

where

$$
\rho_{T(S)}\left(E, V_{S}\right)=\frac{1}{2 \pi} \frac{\Gamma_{T(S)}}{\left[E-\varepsilon_{0}\left(V_{S}\right)\right]^{2}+\left[\left(\Gamma_{T}+\Gamma_{S}\right) / 2\right]^{2}}
$$

is the partial density of states of the LUMO resonance, positioned at $\varepsilon_{0}\left(V_{S}\right)$, due to the coupling $\Gamma_{T(S)}$ to the tip (sample) electrode (neglecting energy dependence in $\left.\Gamma_{T(S)}\right)$. We take the equilibrium Fermi energy $\varepsilon_{F}=0$ as the energy reference and define the tip and surface chemical potentials as $\mu_{T}=V_{S} / 2$ and $\mu_{S}=-V_{S} / 2$, respectively. The resonance parameters $\left\{\varepsilon_{0}, \Gamma_{T}, \Gamma_{S}\right\}$ are readily fitted to the DFT-NEGF calculations as a function of $\mathrm{C}_{60}$-position and voltage, as shown in Fig. 3(b).

\section{VOLTAGE DROP}

Remarkably, the nonequilibrium electronic structure reveals a strong variation of $\varepsilon_{0}$ with $\mathrm{C}_{60}$-position for positive sample voltages. This is a central finding of this work and below we shall show that it can explain the strong polarity dependence of the TLF seen in the experiments. In Fig. 3(c) we illustrate this by plotting the change in $\varepsilon_{0}$ relative to $\mu_{S}$, as a function of $\mu_{T}$ for the various $\mathrm{C}_{60}$-displacements. For $\mu_{T}<0$ the $\varepsilon_{0}$ mainly follows $\mu_{S}$, while for $\mu_{T}>0$ a small increase in $d_{1}$ and thus coupling to the tip, makes $\varepsilon_{0}$ follow $\mu_{T}$ rather than $\mu_{S}$, despite $\Gamma_{S}>\Gamma_{T}$.

The voltage dependence of $\varepsilon_{0}$, or equivalently the voltage profile across the junction, can be understood roughly as a disposition of the system to maintain a constant electron charge $Q$ in the resonance. ${ }^{46}$ In order to illustrate this we consider a simple model calculation. Within the resonance model the LUMO charge is given by

$$
Q\left(V_{S}\right)=\int_{-\infty}^{\mu_{T}} \rho_{T}\left(E, V_{S}\right) d E+\int_{-\infty}^{\mu_{S}} \rho_{S}\left(E, V_{S}\right) d E
$$



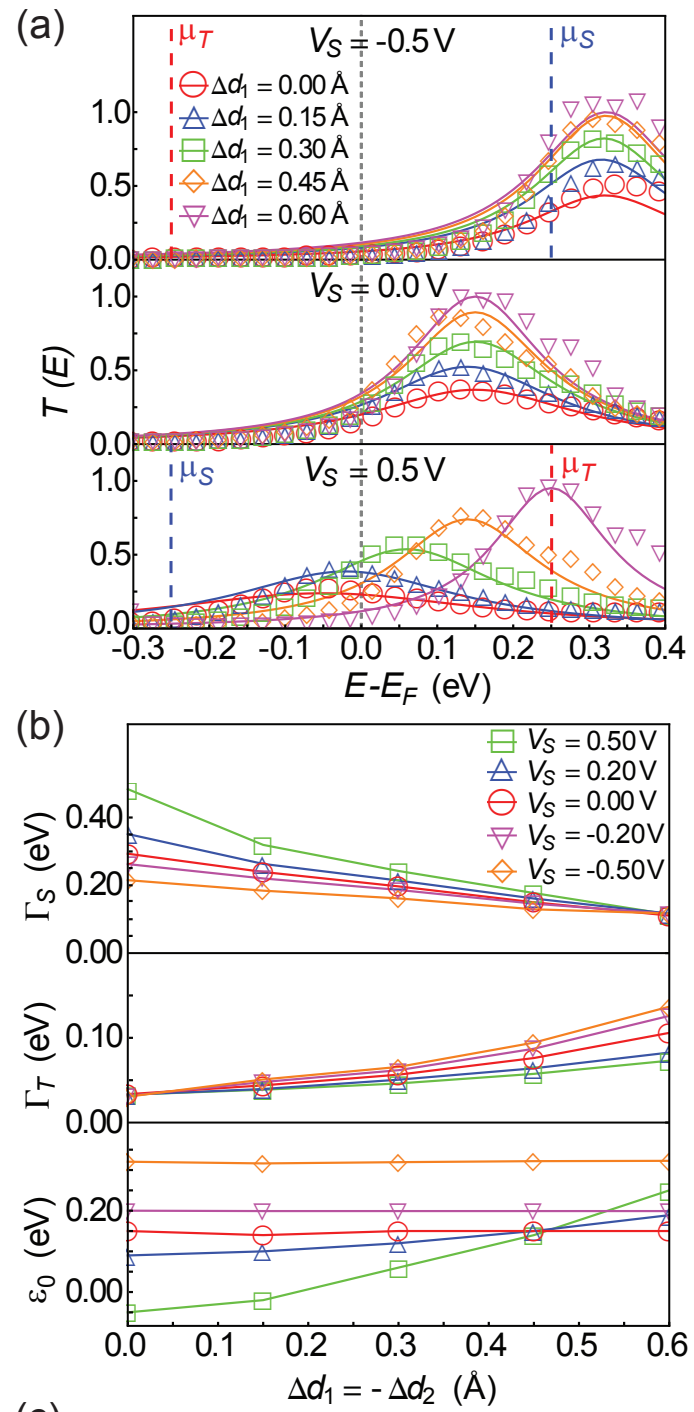

(c)

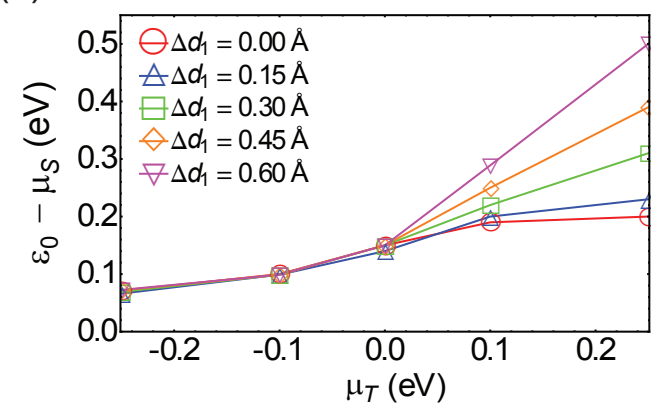

FIG. 3: (color online) (a) Transmission spectra $T\left(E, V_{S}\right)$ for different sample bias voltage $V_{S}$ as the $\mathrm{C}_{60}$-surface bond length is increased by $\Delta d_{1}$. Data points correspond to DFTNEGF simulations and solid lines are fits to the Breit-Wigner formula [Eq. [1]] for the transmission through a single molecular orbital. (b) Fitted LUMO level position $\epsilon_{0}$ and coupling functions $\Gamma_{S}$ and $\Gamma_{T}$ due to the surface and tip coupling, respectively, and (c) voltage drop across the $\mathrm{C}_{60}$-surface interface as a function of $\mu_{T}$ and $\Delta d_{1}$. The solid lines in (b-c) are guides to the eye. (a)

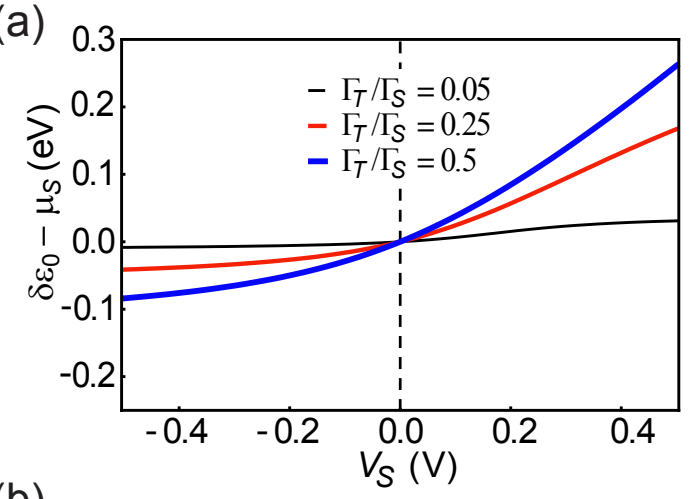

(b)

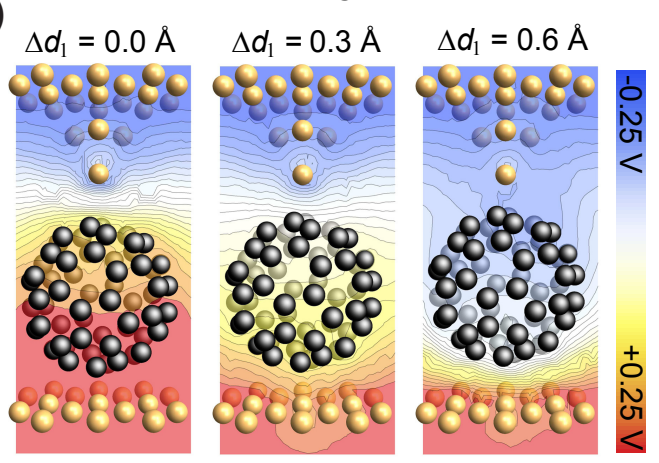

FIG. 4: (color online) (a) Simple model calculation of the change in resonance position assuming charge-neutrality of the resonance at finite bias. We use $\varepsilon_{0}=0.1 \mathrm{eV}, \Gamma_{S}+\Gamma_{T}=$ $0.2 \mathrm{eV}$ (fixed), and vary $\Gamma_{T} / \Gamma_{S}$ (distance to tip). The main voltage drop occurs between tip and $\mathrm{C}_{60}$ (resonance) for $V_{S}<$ 0 and small tip coupling, while it shifts to the $\mathrm{C}_{60}$-substrate interface for $V_{S}>0$ and stronger tip coupling. (b) Voltage drop (change in DFT one-electron potential) calculated for increasing $\mathrm{C}_{60}$-surface bond length at a positive sample bias of $0.5 \mathrm{~V}$. The voltage difference between consecutive contour lines is $18 \mathrm{mV}$ (shown in a plane through the tip atom).

If we assume constant LUMO charge independent of the applied bias, i.e., $Q\left(V_{S}\right)=Q(0)$, we may determine the bias-dependent change in LUMO position, $\delta \varepsilon_{0}\left(V_{S}\right)$ from Eq. (3). To mimic the change in $\mathrm{C}_{60}$-tip distance, $d_{2}$, for fixed electrode distance, $L$, we vary $\Gamma_{T} / \Gamma_{S}$ for fixed $\Gamma_{T}+\Gamma_{S}$. In Fig. 4(a) it is seen how this simplified model reproduces the cross-over in the full DFT calculation [Fig. 3(c)] for positive sample voltage when the contact is formed. Thus the main voltage drop changes from being between tip and $\mathrm{C}_{60}$ for $V_{S}<0$ to being between surface and $\mathrm{C}_{60}$ when $V_{S}>0$ and the distance to the tip is decreased $\left(\left|\delta \varepsilon_{0}-\mu_{S}\right|>\left|\delta \varepsilon_{0}-\mu_{T}\right|\right)$. From Eq. (3) we can thus infer that in nonequilibrium there is a sensitive balance between coupling strengths $\left(\Gamma_{T / S}\right)$ and electrode chemical potentials $\left(\mu_{T / S}\right)$ that can displace the voltage drop from one interface to the other with a small relative change in coupling strengths.

The voltage drop effect can also be seen directly in the actual voltage drop landscape (change in the oneelectron potential with respect to equilibrium) shown in 
Fig. 4(b). The voltage drop is observed to shift from the $\mathrm{C}_{60}$-tip interface to the $\mathrm{C}_{60}$-substrate interface with a small $\mathrm{C}_{60}$-displacement, an effect not present for $V_{S}<0$ (not shown).

\section{HEATING AND FLUCTUATIONS}

We next explain how the strong variation of $\varepsilon_{0}$ with $\mathrm{C}_{60}$-position for $V_{S}>0$ can be related to the strong polarity dependence of the TLF. We start by assuming that the main current-dependence comes from the excitation of $\mathrm{C}_{60}$ CM-motion, described by a harmonic potential with $\hbar \Omega \approx 4 \mathrm{meV}$ [cf. Fig. 2(a) at $L_{0}$ ]. Guided by the fact that the switching rates observed in the experiments (ms time scale) are very slow compared to CM oscillations, we propose that the switching involves a slow "bottle-neck" process, possibly involving tunneling along the reaction coordinate $(\mathrm{RC})$, and that this process takes place when the excursion of the $\mathrm{C}_{60}\left(\Delta d_{1}\right)$ is beyond some critical distance from the equilibrium position. Inspired by the study of tunneling of a $\mathrm{C}_{60}$ molecule in the lowconductance regime ${ }^{\sqrt{14}}$ we express the switching rate as

$$
R\left(V_{S}\right)=r_{s} e^{-d_{c}^{2} /\left\langle\Delta d_{1}\left(V_{S}\right)^{2}\right\rangle}=r_{s} e^{-\Delta /\left\langle E_{\mathrm{CM}}\left(V_{S}\right)\right\rangle},
$$

i.e., as a product of the probability of $\mathrm{C}_{60}$ being at an excursion $\Delta d_{1}=d_{c}$ away from equilibrium and of a rate, $r_{s}$, describing the slow process along the RC. The critical distance $d_{c}$, or equivalently the energy barrier $\Delta$, controls how far the $\mathrm{C}_{60}$ needs to move in order to facilitate switching. The mean displacement $\left\langle\Delta d_{1}^{2}\right\rangle$, or equivalently the mean oscillator energy $\left\langle E_{\mathrm{CM}}\left(V_{S}\right)\right\rangle$, are quantities which we can calculate within our TLF-model.

The excitation of the $\mathrm{C}_{60}$-CM motion by the current is determined from the electronic coupling to this motion. Using Fig. 3(b) we extract the electron-vibration coupling $M$ from the shift in resonance position with $\mathrm{C}_{60^{-}}$ displacement $d_{1}$ via ${ }^{47}$

$$
M\left(V_{S}\right) \approx \frac{l_{0}}{\sqrt{2}} \partial_{d_{1}} \varepsilon_{0}\left(V_{S}\right)
$$

We evaluate the slope, $\partial_{d_{1}} \varepsilon_{0}\left(V_{S}\right)$, around $\Delta d_{1}=0.3 \AA$, which is in the middle of the transition region [Fig. 3(b)], and note that the slope does not change significantly as we increase $\Delta d_{1}$. The characteristic oscillator length is $l_{0}=\sqrt{\hbar / m \Omega} \approx 0.04 \AA\left(\mathrm{C}_{60}\right.$ mass $\left.m\right)$, which is comparable to the size of the transition region in Fig. 2. The extracted electron-vibration coupling, $M$, is shown in Fig. 5 as a function of sample voltage. A remarkably strong enhancement is evident for $V_{S}>0$.

The excitation of the CM-motion, as seen in its mean energy $\left\langle E_{\mathrm{CM}}\left(V_{S}\right)\right\rangle$, can be obtained from the biasdependent rates of phonon emission, $\gamma_{\mathrm{em}}\left(V_{S}\right)$, and of electron-hole pair generation, $\gamma_{\mathrm{eh}}\left(V_{S}\right)$. These rates can be determined within first order perturbation theory
(Fermi's Golden rule). Since $\hbar \Omega$ is much smaller than all other electronic parameters, we may write

$$
\begin{aligned}
\gamma_{\mathrm{em}}\left(V_{S}\right) & \approx \frac{4 \pi}{\hbar}\left|M\left(V_{S}\right)\right|^{2} \theta\left(\frac{e\left|V_{S}\right|}{\hbar \Omega}-1\right) \\
& \times \int_{-\left|V_{S}\right| / 2}^{\left|V_{S}\right| / 2} \rho_{S}\left(E, V_{S}\right) \rho_{T}\left(E, V_{S}\right) d E, \\
\gamma_{\mathrm{eh}}\left(V_{S}\right) & \approx 4 \pi\left|M\left(V_{S}\right)\right|^{2} \Omega \\
& \times\left[\rho\left(\mu_{T}, V_{S}\right) \rho_{T}\left(\mu_{T}, V_{S}\right)+\rho\left(\mu_{S}, V_{S}\right) \rho_{S}\left(\mu_{S}, V_{S}\right)\right],
\end{aligned}
$$

where $\rho=\rho_{T}+\rho_{S}$. From these rates we can write a rate equation for the mean phonon occupation $\left\langle n\left(V_{S}\right)\right\rangle$,

$$
\left\langle\dot{n}\left(V_{S}\right)\right\rangle=\gamma_{\mathrm{em}}\left(V_{S}\right)-\left\{\gamma_{\mathrm{eh}}\left(V_{S}\right)+\gamma_{\mathrm{ph}}\right\}\left\{\left\langle n\left(V_{S}\right)\right\rangle-n_{B}\right\},
$$

where $\gamma_{\mathrm{ph}}$ represents the vibrational relaxation due to anharmonic coupling to phonons in tip/substrate and $n_{B}$ is the Bose-Einstein (equilibrium) phonon occupation of the considered mode. The steady-state solution is simply

$$
\left\langle n\left(V_{S}\right)\right\rangle=n_{B}+\frac{\gamma_{\mathrm{em}}\left(V_{S}\right)}{\gamma_{\mathrm{eh}}\left(V_{S}\right)+\gamma_{\mathrm{ph}}} .
$$

Following Refs. 47/48 one can estimate a phonon damping to the substrate of $\mathrm{C}_{60}$-CM motion via the formula

$$
\gamma_{\mathrm{ph}}=\frac{m}{m_{\mathrm{Cu}}} \frac{3 \pi}{2 \omega_{e}^{3}} \Omega^{4} \approx 0.1 \Omega
$$

where $m_{\mathrm{Cu}}$ is the mass of a substrate atom, and $\omega_{e}=30$ $\mathrm{meV}$ a frequency characterizing the elastic response. This damping is likely to be exaggerated compared to the experimental situation since the $\mathrm{C}_{60}$ is adsorbed on a reconstructed surface with low-coordinated surface atoms and lower density of long wavelength phonons. This is a critical point for the explanation of the experimental result. We find that the best agreement is obtained for $\gamma_{\mathrm{ph}} \approx 0.001 \Omega$. In Fig. 6 we show how $\gamma_{\mathrm{em}}, \gamma_{\mathrm{eh}}$, and $\langle n\rangle$ varies with the sample voltage $V_{S}$ along with the effective temperature defined through a Bose-Einstein distribution $\left\langle n\left(V_{S}\right)\right\rangle=1 /\left(e^{\hbar \Omega / k_{B} T_{\text {eff }}\left(V_{S}\right)}-1\right)$. In all cases we see an enhancement for $V_{S}>0$. If we use $\gamma_{\mathrm{ph}} \approx 0.1 \Omega$ the mean occupation and effective temperature become a factor 100 smaller, but exhibit the same behavior as in Figs. 6(c)-(d). Finally we can calculate the oscillator energy as $\left\langle E_{\mathrm{CM}}\left(V_{S}\right)\right\rangle=\hbar \Omega\left(\left\langle n\left(V_{S}\right)\right\rangle+1 / 2\right)$ and thus the current-dependent rate from Eq. (4).

Fig. 7(a) shows how the calculated switching yield $Y\left(V_{S}\right)$ (blue squares), defined as the switching rate per tunneling electron, can reproduce the experimental data (red circles) if we use a barrier height of $\Delta=2 \hbar \Omega$, a "tunnel-rate" $r_{s}=80 \mathrm{~ms}^{-1}, \gamma_{\mathrm{ph}} \sim 0.001 \Omega$, and a background temperature of $T=14 \mathrm{~K}$ as fitting parameters. The slightly elevated temperature, compared to the experiment performed at $T=7 \mathrm{~K}$, helps to smoothen the onset of the switching rate at small voltages. This can be justified by vibrational heating of other modes and their anharmonic coupling to the $\mathrm{CM}$ motion of the $\mathrm{C}_{60}$. The relatively slow $r_{s}$ is consistent with a tunneling process, 


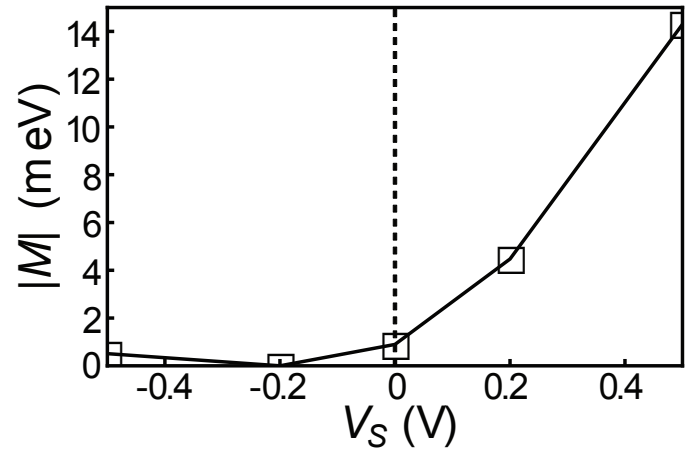

FIG. 5: (color online) Calculated electron-vibration coupling $|M|$ for the $\mathrm{C}_{60} \mathrm{CM}$ motion (black squares) as a function of sample voltage $V_{S}$, based on fits to the LUMO resonance in the transition region at the electrode separation $L_{0}$.

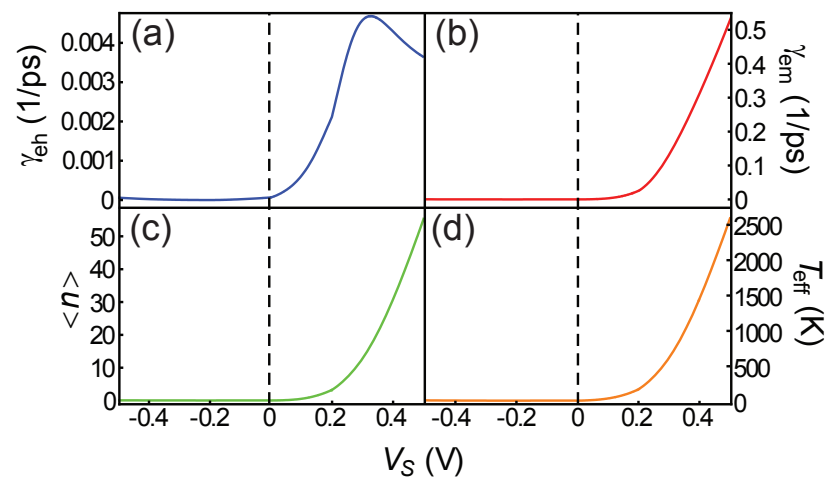

FIG. 6: (color online) Rates of (a) electron-hole pair generation, (b) phonon emission, and resulting (c) mean phonon occupation as a function of sample bias for $\gamma_{\mathrm{ph}} \sim 0.001 \Omega$. (d) Effective temperature $T_{\text {eff }}\left(V_{S}\right)$ corresponding to the mean phonon occupation in (c).

and the small $\Delta$ is consistent with the fact that we could not determine the barrier with our DFT calculations.

In Fig. 7(b) we show the calculated fluctuation rate in the case of constant zero-bias electron-vibration coupling where only the spectral energy-dependence of the molecule are considered, cf. the explanation presented in Ref. 25] for the polarity dependence. However, it is clear that we are only able to reproduce the experimental results if we take the behaviour of the electronvibration matrix element with bias into account. These findings suggests that (i) the strong polarity dependence of the switching is rooted in the nonequilibrium electronvibration coupling in the transition region where the bond-formation between tip and $\mathrm{C}_{60}$ takes place, and (ii) that the reason for the observed saturation of the switching rate per electron is due to the steadily increasing electron-hole pair damping with bias, Eq. (8), so this becomes comparable with $\gamma_{\mathrm{ph}}$. This is an impor-

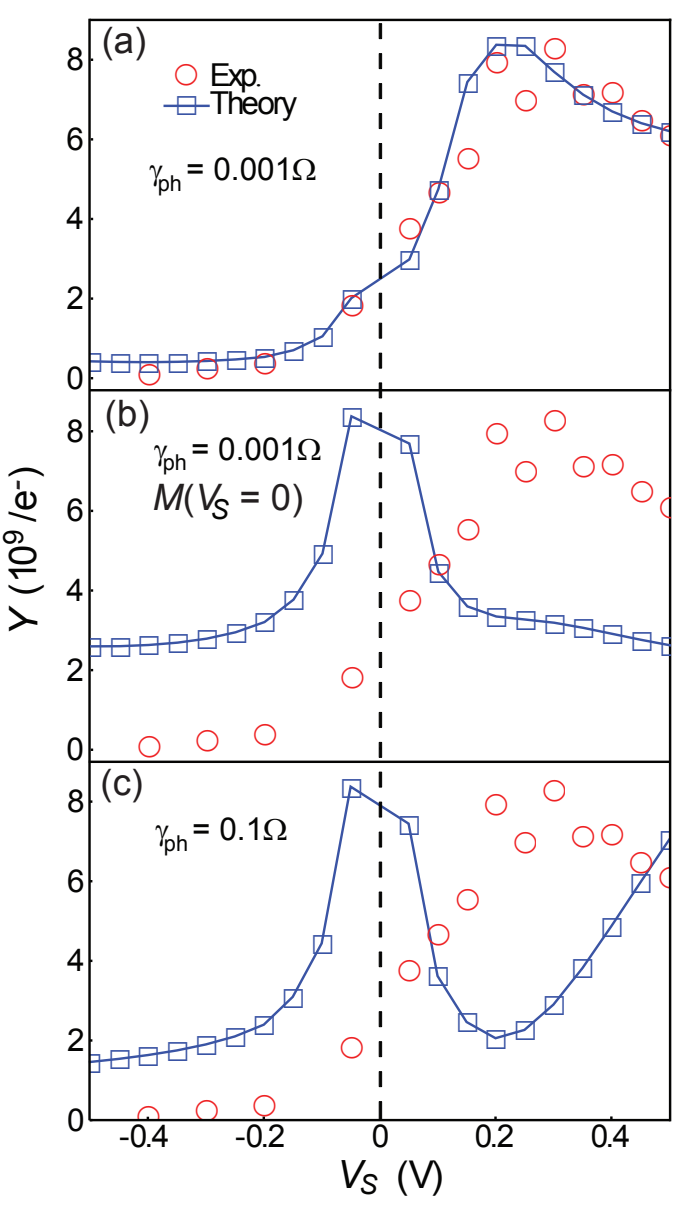

FIG. 7: (color online) (a) Calculated switching yield $Y\left(V_{S}\right)$ (blue squares) based on the excitation of the $\mathrm{C}_{60} \mathrm{CM}$-motion $(\hbar \Omega=4 \mathrm{meV})$ using Eq. (4) with $r_{s}=80 \mathrm{~ms}^{-1}, T=14 \mathrm{~K}$, and $\Delta=2 \hbar \Omega$. (b) Switching yield in the case of constant electron-vibration coupling, $M\left(V_{S}\right)=M\left(V_{S}=0\right)$. (c) Same as (a) but with $\gamma_{\mathrm{ph}} \sim 0.1 \Omega \ll \gamma_{\mathrm{eh}}$. For comparison also the experimental data from Ref. 25] are shown (red circles).

tant point as illustrated in Fig. 7(c) where we show how the switching yield using the estimate $\gamma_{\mathrm{ph}}=0.1 \Omega$ grows for $V_{S}>0.2 \mathrm{~V}$ (contrary to the experiment).

We note that the calculated current is roughly linear in voltage as in the experiments, and thus do not contribute significantly to the polarity dependence of the switching compared to the pronounced effect seen in Fig. 5 for the electron-vibration coupling $M$. We further note that one theoretical study ${ }^{49}$ has previously reported a nonlinear, polarity-independent $M$ for a smaller symmetric molecular junction and only at significantly higher voltages $V>0.4 \mathrm{~V}$. 


\section{EFFECT OF CURRENT-INDUCED FORCE}

In this section we estimate the change in the potential energy landscape in Fig. 1(b) when a nonequilibrium force is exerted on the $\mathrm{C}_{60}$-tip bond during contact formation. To calculate this additional bond force we consider the interaction between the $\mathrm{C}_{60}$ LUMO resonance and a wide band centered on the tip (see inset in Fig. 8). For this system we define the following two-site Hamiltonian

$$
\mathbf{H}=\left(\begin{array}{cc}
\varepsilon_{0}\left(d_{2}, V_{S}\right) & t\left(d_{2}, V_{S}\right) \\
t\left(d_{2}, V_{S}\right) & \mu_{T}\left(V_{S}\right)
\end{array}\right),
$$

where we explicitly stress the dependence on bias and bond length $d_{2}$. The interaction strength $t\left(d_{2}, V_{S}\right)$ is calculated using $\Gamma_{T}\left(d_{2}, V_{S}\right)=2 \pi \gamma_{T}\left|t\left(d_{2}, V_{S}\right)\right|^{2}$, where $\gamma_{T}$ is the wide band density of states on the tip, i.e., a constant which can be fitted to reproduce the transmission spectra in Fig. 3(a). The bond force is calculated using the general expression 50

$$
\begin{aligned}
F_{b}\left(d_{2}, V_{S}\right) & =-2 \operatorname{Tr}\left[\left(\partial_{d_{2}} \mathbf{H}\right) \mathbf{D}\right] \\
& =-2\left[D_{11} \partial_{d_{2}} \varepsilon_{0}\left(d_{2}, V_{S}\right)+2 D_{12} \partial_{d_{2}} t\left(d_{2}, V_{S}\right)\right],
\end{aligned}
$$

where a factor of 2 is included to account for spin. The elements, $D_{11}$ and $D_{12}\left(=D_{21}\right)$ of the density matrix $\mathbf{D}$ are determined from the spectral properties of the considered states, 51 which can be calculated from the fits in Fig. 3(b).

Since we only consider motion along a single coordinate the current-induced force is energy conserving, $\delta F_{b}\left(d_{2}, V_{S}\right)=F_{b}\left(d_{2}, V_{S}\right)-F_{b}\left(d_{2}, 0\right)$, enabling us to calculate the change in bond energy at a given bias,

$$
\Delta E_{b}\left(d_{2}, V_{S}\right)=-\int_{d_{2, i}}^{d_{2}} \delta F_{b}\left(z, V_{S}\right) d z
$$

The integration limits are defined such that initially a contact is established, $d_{2, i} \approx 5.6 \AA \mathrm{cf}$. the equilibrium curve in Fig. 8, and then we integrate along a path where the $\mathrm{C}_{60}$-tip contact is gradually separated. Addition of the energy term in Eq. (13) on top of the equilibrium total energy in Fig. 11(b) yields the bias-corrected curves shown in Fig. 8. Astoundingly, we see that only at positive $V_{S}$ a tiny barrier of the order a few meV may appear between two stable configurations corresponding to contact and tunneling cases, respectively. The origin of the significant lowering of the tunneling part of the binding energy curve for positive $V_{S}$ is related to the asymmetry in resonance position, which yields a large contribution from $\partial_{d_{2}} \varepsilon_{0}$ in Eq. 12 only at positive $V_{S}$. Finally, we note that the order of magnitude of the nonequilibrium barrier is in accordance with our assumption in the fluctuation calculation in Fig. 7 .

\section{CONCLUSIONS}

In summary, we have presented the results of firstprinciples calculations which combined with a heating

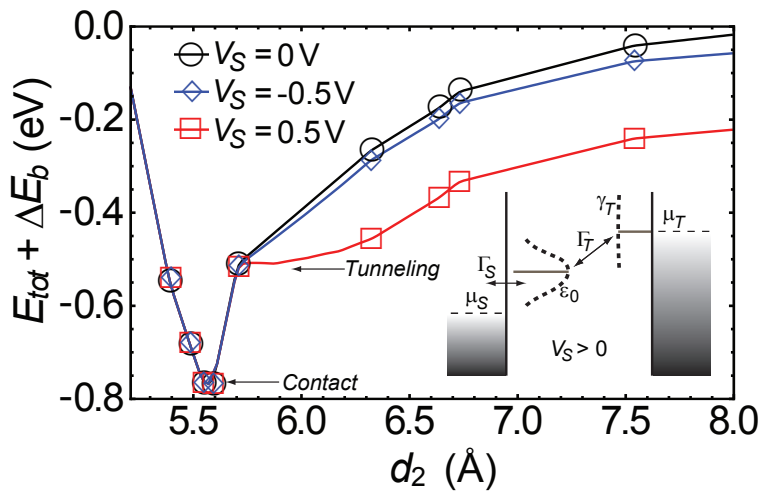

FIG. 8: (color online) Equilibrium DFT total energy from Fig. 1. b) plus an additional contribution due to a (conservative) current-induced force. Note that energy is here plotted against $\mathrm{C}_{60}$-tip bond length $d_{2}$ instead of electrode separation. Inset: Simple model describing a bond between the molecular resonance at $\epsilon_{0}$ and a wide band centered on the tip, which follows the chemical potential $\mu_{T}$.

model and assuming a small energy barrier can explain the experimentally observed bias-dependent TLF observed in a $\mathrm{C}_{60} \mathrm{STM}$ junction. Our main point is that the electron-vibration coupling can depend very strongly on the bias polarity. In this system we can trace this back to sensitivity of the nonequilibrium electronic structure/voltage drop with respect to the $\mathrm{C}_{60}$-motion just when the contact is being formed. The bias dependence of the electron-vibration coupling has so far not been considered in most calculations of inelastic electron transport and current-induced excitations. It remains to be answered to what extend this is important in general. In order to model the experimental switching we had to assume a small energy barrier for the $\mathrm{C}_{60}$-motion at the contact formation point. Although it is likely that the small barrier is masked by inaccuracy inherent in the DFT, the finite unit-cell employed, or numerical error, we showed that the nonequilibrium can induce significant changes in the potential energy surface. Our estimate of the current-induced force exerted on the $\mathrm{C}_{60}$-tip bond did indeed indicate an energy barrier for positive sample voltage.

In the presence of a significant current a number of different excitation mechanisms can become active. Recently, it has been discussed how current-induced forces can lead to "run-away" instabilities such as bond-rupture for highly conducting systems $G \sim G_{0}$, and voltages in the range involved in the present experiment 52153 . TLF experiments seems to be a promising way to probe these. The runaway effect requires the action of several vibration modes and we have limited our discussion here to a single mode. Our results demonstrate how the full nonequilibrium electronic structure can be of crucial importance for the formation/breaking of chemical bonds and electron-vibration coupling in the presence of cur- 
rent.

\section{Acknowledgments}

We are grateful to Richard Berndt, Jörg Kröger, and Nicolás Néel for stimulating discussions and comments on an early version of our manuscript. We are also thankful to Hiromu Ueba for valuable suggestions. We also acknowledge computer resources from the DCSC.
* Present address: Department of Physics and Astronomy, Interdisciplinary Nanoscience Center, Aarhus University, 8000 Aarhus C, Denmark

1 M. Galperin, M. A. Ratner, A. Nitzan, and A. Troisi, Science 319, 1056 (2008).

2 N. Agraït, J. Rodrigo, and S. Viera, Phys. Rev. B 47, $12345(1993)$

3 Z. Donhauser, B. Mantooth, K. Kelly, L. Bumm, J. Monnell, J. Stapleton, D. Price, A. Rawlett, D. Allara, J. Tour, et al., Science 292, 2303 (2001).

${ }^{4}$ R. Wassel, R. Fuierer, N. Kim, and C. Gorman, Nano Lett. 3, 1617 (2003).

${ }^{5}$ W. H. A. Thijssen, D. Djukic, A. F. Otte, R. H. Bremmer, and J. M. van Ruitenbeek, Phys. Rev. Lett. 97, 226806 (2006).

6 A. Sperl, J. Kröger, and R. Berndt, Phys. Rev. B 81, 035406 (2010).

7 S. J. v. d. Molen and P. Liljeroth, J. Phys.: Condens. Matter 22, 133001 (2010).

8 D. M. Eigler, C. P. Lutz, and W. E. Rudge, Nature 352, 600 (1991).

9 J. A. Stroscio and R. J. Celotta, Science 306, 242 (2004).

10 B. C. Stipe, M. A. Rezaei, and W. Ho, Phys. Rev. Lett. 81, 1263 (1998).

11 B.-Y. Choi, S.-J. Kahng, S. Kim, H. Kim, H. W. Kim, Y. J. Song, J. Ihm, and Y. Kuk, Phys. Rev. Lett. 96, 156106 (2006).

12 J. Henzl, M. Mehlhorn, H. Gawronski, K.-H. Rieder, and K. Morgenstern, Angew. Chem. Int. Ed. 45, 603 (2006).

13 P. Liljeroth, J. Repp, and G. Meyer, Science 317, 1203 (2007)

14 A. V. Danilov, P. Hedegård, D. S. Golubev, T. Bjørnholm, and S. E. Kubatkin, Nano Lett. 8, 2393 (2008).

15 A. Halbritter, P. Makk, S. Csonka, and G. Mihaly, Phys. Rev. B 77, 075402 (2008).

16 M. L. Trouwborst, E. H. Huisman, S. J. van der Molen, and B. J. van Wees, Phys. Rev. B 80, 081407 (2009).

17 T. Kumagai, M. Kaizu, H. Okuyama, S. Hatta, T. Aruga, I. Hamada, and Y. Morikawa, Phys. Rev. B 79, 035423 (2009).

18 F. Mohn, J. Repp, L. Gross, G. Meyer, M. S. Dyer, and M. Persson, Phys. Rev. Lett. 105, 266102 (2010).

19 R. Ohmann, L. Vitali, and K. Kern, Nano Lett. 10, 2995 (2010).

20 T. Brumme, O. A. Neucheva, C. Toher, R. Gutierrez, C. Weiss, R. Temirov, A. Greuling, M. Kaczmarski, M. Rohlfing, F. S. Tautz, et al., Phys. Rev. B 84, 115449 (2011).

21 T. Huang, J. Zhao, M. Feng, A. A. Popov, S. Yang, L. Dunsch, and H. Petek, Nano Lett. 11, 5327 (2011).

22 T. Kumagai, A. Shiotari, H. Okuyama, S. Hatta, T. Aruga, I. Hamada, T. Frederiksen, and H. Ueba, Nature Mater.
11, 167 (2012).

${ }^{23}$ H. Park, J. Park, A. K. L. Lim, E. H. Anderson, A. P. Alivisatos, and P. L. McEuen, Nature 407, 57 (2000).

24 G. Schulze, K. J. Franke, A. Gagliardi, G. Romano, C. S. Lin, A. L. Rosa, T. A. Niehaus, T. Frauenheim, A. D. Carlo, A. Pecchia, et al., Phys. Rev. Lett. 100, 136801 (2008)

25 N. Néel, J. Kröger, and R. Berndt, Nano Lett. 11, 3593 (2011).

26 J. A. Larsson, S. D. Elliott, J. C. Greer, J. Repp, G. Meyer, and R. Allenspach, Phys. Rev. B 77, 115434 (2008).

27 N. Néel, J. Kröger, L. Limot, and R. Berndt, Nano Lett. 8, 1291 (2008).

28 N. Néel, J. Kröger, L. Limot, T. Frederiksen, M. Brandbyge, and R. Berndt, Phys. Rev. Lett. 98, 065502 (2007).

29 G. Schull, T. Frederiksen, M. Brandbyge, and R. Berndt, Phys. Rev. Lett. 103, 206803 (2009).

${ }^{30}$ G. Schull, T. Frederiksen, A. Arnau, D. Sanchez-Portal, and R. Berndt, Nat. Nanotechnol. 6, 23 (2011).

31 G. Schull, Y. J. Dappe, C. González, H. Bulou, and R. Berndt, Nano Lett. 11, 3142 (2011).

32 J. M. Soler, E. Artacho, J. D. Gale, A. Garcia, J. Junquera, P. Ordejon, and D. Sanchez-Portal, J. Phys.: Condens. Matter 14, 2745 (2002).

33 M. Brandbyge, J.-L. Mozos, P. Ordejon, J. Taylor, and K. Stokbro, Phys. Rev. B 65, 165401 (2002).

34 J. P. Perdew, K. Burke, and M. Ernzerhof, Phys. Rev. Lett. 77, 3865 (1996).

35 S. Garcia-Gil, A. Garcia, N. Lorente, and P. Ordejon, Phys. Rev. B 79, 075441 (2009).

36 S. F. Boys and F. Bernardi, Mol. Phys. 19, 553 (1970).

37 G. Kresse and J. Furthmüller, Phys. Rev. B 54, 11169 (1996).

38 I. D. Hands, J. L. Dunn, and C. A. Bates, Phys. Rev. B 81, 205440 (2010).

39 B. W. Heinrich, M. V. Rastei, D.-J. Choi, T. Frederiksen, and L. Limot, Phys. Rev. Lett. 107, 246801 (2011).

40 I. Hamada, A. Masaaki, and M. Tsukada, Phys. Rev. B 85, 121401(R) (2012).

41 M. Vanin, J. J. Mortensen, A. K. Kelkkanen, J. M. GarciaLastra, K. S.Thygesen, and K. W. Jacobsen, Phys. Rev. B 81, 081408(R) (2010).

42 J. Wellendorff, K. T. Lundgaard, A. Møgelhøj, V. Petzold, D. D. Landis, J. K. Nørskov, T. Bligaard, and K. W. Jacobsen, Phys. Rev. B 85, 235149 (2012).

43 A. A. Dzhioev and D. S. Kosov, J. Chem. Phys 135, 074701 (2011)

44 S. Datta, Electronic transport in mesoscopic systems (Cambridge University Press, 1995).

45 F. D. Novaes, M. Cobian, A. Garcia, P. Ordejon, H. Ueba, and N. Lorente, arXiv:1101.3714v1 (2011).

46 M. Brandbyge, N. Kobayashi, and M. Tsukada, Phys. Rev. 
B 60, 17064 (1999)

47 S. Gao, M. Persson, and B. Lundqvist, Phys. Rev. B 55, 4825 (1997).

48 J. Leiro and M. Persson, Surf. Science 207, 473 (1989).

49 N. Sergueev, D. Roubtsov, and H. Guo, Phys. Rev. Lett. 95, 146803 (2005).

50 T. N. Todorov, J. Hoekstra, and A. P. Sutton, Philos. Mag. B 80, 421 (2000).
51 M. Brandbyge, K. Stokbro, J. Taylor, J.-L. Mozos, and P. Ordejon, Phys. Rev. B 67, 193104 (2003).

52 D. Dundas, E. J. McEniry, and T. N. Todorov, Nat. Nanotechnol. 4, 99 (2009).

53 J.-T. Lu, M. Brandbyge, and P. Hedegard, Nano Lett. 10, 1657 (2010). 